\title{
Numerical Investigation of Self-Starting Capability of Vertical-Axis Wind Turbines at Low Reynolds Numbers
}

\author{
Hsieh-Chen Tsai* and Tim Colonius ${ }^{\dagger}$ \\ California Institute of Technology, Pasadena, CA 91125, USA
}

\begin{abstract}
The self-starting capability of a NACA 0018 multi-bladed vertical-axis wind turbine is numerically investigated. The immersed boundary method is used to simulate the flow around a two-dimensional cross section of the wind turbine and the predictor-corrector method is used to couple the equation of motion of the turbine. A simple load model, which is linearly proportional to turbine angular velocity, is used for the load of the turbine. The angular velocity is characterized as a function of Reynolds number, density ratio, and viscous coefficient of the proposed load model. The power outputs and moment coefficients of motor-driven and flow-driven vertical-axis wind turbine are compared. For a particular Reynolds number, as the load on the flow-driven turbine is increased, the tip speed is reduced until the turbine fails to coherently rotate. The flow-driven and motordriven moment coefficients in the computation have good agreement between each other and are qualitatively similar to the torque measured in experiments. ${ }^{1}$ These computations suggest that the load of a flow-driven turbine can be well-represented by the proposed load model and a motor-driven turbine can reproduce the physics of a flow-driven turbine within the range of tip-speed ratio examined. A simple model is proposed in order to analyze the starting torque. By assuming that the inertia of the blade is much larger than the fluid, the turbine can be considered stationary in the flow. The starting torque distribution of a multi-bladed turbine indicates the important orientations corresponding to maximum torque generation, at which a self-starting turbine always starts, and a stable equilibrium, where a non-self-starting turbine oscillates. These features agree with observations from the full simulations of the starting process. We further model the starting torque distribution by considering a single blade at different orientations, and construct starting torque distributions for multi-bladed turbines by linearly combining the torques at the respective positions of the blades. We show that this approximation is valid for a sufficiently low turbine solidity of about 0.5. Using this model, we find optimal starting configuration for a multi-bladed low-solidity vertical-axis wind turbine.
\end{abstract}

\section{Nomenclature}

$\begin{array}{ll}c & \text { airfoil chord length } \\ C_{M} & \text { moment coefficient } \\ C_{P} & \text { power coefficient } \\ F & \text { friction coefficient of the load } \\ I_{O} & \text { moment of inertia of the blade about the rotation center of the VAWT } \\ N_{b} & \text { number of blades } \\ O & \text { turbine rotation center } \\ R & \text { radius of the turbine } \\ R e & \text { Reynolds Number } \\ t^{*} & \text { convective time units } \\ U_{\infty} & \text { freestream velocity } \\ V & \text { volume of the blade }\end{array}$

${ }^{*}$ Graduate Student, Mechanical Engineering, Student Member AIAA

${ }^{\dagger}$ Professor, Mechanical Engineering, Associate Fellow AIAA 


$\begin{array}{ll}\mathbf{x} & \text { position vector from } O \text { to a point in the blade } \\ \Delta t & \text { time step } \\ \Delta x & \text { grid spacing } \\ \lambda & \text { tip-speed ratio } \\ \nu & \text { dynamic viscosity } \\ \rho & \text { fluid density } \\ \rho_{b}(\mathbf{x}) & \text { density of the blade at position } \mathbf{x} \\ \rho_{e} & \text { effective density } \\ \Omega & \text { angular velocity } \\ \phi_{\rho} & \text { density ratio } \\ \psi & \text { static pitch angle } \\ \sigma & \text { turbine solidity } \\ \tau_{f b} & \text { moment exerted by the fluid about the turbine rotation center } \\ \tau_{L} & \text { torque generated by a load } \\ \theta & \text { azimuthal angle } \\ \theta_{0} & \text { initial azimuthal angle }\end{array}$

\section{Subscripts}

avg averaged value

opt optimal value

\section{Introduction}

Compared to horizontal designs lift-based (Darrieus) vertical-axis wind turbines (VAWT) offer several advantages including low noise, accessibility, and omni-directionality. However, they also suffer disadvantages like a lower-efficiency, high cyclic loadings on the supporting structure and the blades, and difficult-toself-starting. Researchers like Kirke ${ }^{2}$ and Pawsey ${ }^{3}$ has widely discussed and well summarized the relative strength and weakness of vertical and horizontal-axis wind turbines. Since the capability to start reliably and without external assistance under a broad range of wind conditions is crucially important to the development of practical machines, the present work is focused on investigating the self-starting capability of a VAWT numerically at low Reynolds number.

The difficulties associated with self starting of a VAWT are well known. ${ }^{2-5}$ Kirke $^{2}$ have stated that the self-starting of VAWT is possible with a careful selection of blade geometry. Dominy et al. ${ }^{6}$ experimentally showed that a three-bladed VAWT can self-start even using fixed-geometry, symmetric airfoils, but that two-bladed designs can only self-start under particular conditions. Hill et al. ${ }^{5}$ later discussed the physics of self-starting through a simple numerical model and experiments. Experiments showed that in the tip-speed ratio history there exists a plateau region with a tip-speed ratio slightly above unity before the VAWT reaches its operating speed. Based on the forces on a single airfoil fixed at various angles of attack and Reynolds numbers from the experiments done by Sheldahl and Klimas, ${ }^{7}$ the model predicted the net torque over a cycle at various tip-speed ratio and demonstrated two stable equilibria where VAWT can operate. One of the predicted stable equilibria agreed with the plateau region observed in the experiments. However, the model was unable to explain the transition from the plateau region to the final operating condition due to the lack of the interaction between the blades or dynamic stall. Untaroiu et al. ${ }^{8}$ numerically investigated the self-starting capability of VAWT using URANS at $R e \approx 5 \times 10^{5}$. The method has been demonstrated to predict terminal operating speed within $12 \%$ of the measured value. However, the turbine performance during the starting, which is important to the study of self-starting problems, was not accurately captured due to the lack of near-wall and transitional turbulence models.

Several solutions to the starting issue have been suggested regarding the design of the blade. Baker ${ }^{4}$ recommended several blade designs for VAWT that are favorable for self-starting. Beri and Yao ${ }^{9,10}$ numerically investigated the self-starting of VAWT with cambered airfoils and airfoils with modified trailing edge. 
They showed that the VAWT with cambered airfoils has potential to self-start with reduced coefficient of power and the VAWT with tailing-edge-modified airfoils has better performance in self-starting at lower tip-speed ratios with normal coefficient of power. Chen and Kuo ${ }^{11}$ studied the effects of pitch angle and blade camber on the self-starting. The results show that the maximum root-mean-square moment occurs at a pitch angle equal to $5^{\circ}$ outward from the tangential direction for all the blades examined. Moreover, blades with appropriate camber generate a higher root-mean-square moment at all pitch angles.

We previously numerically investigated the effect of Coriolis forces on dynamic stall in VAWT. ${ }^{12}$ As presented in Dunne et al.,$^{13}$ the computational results have a good agreement with experiments performed by Dunne and McKeon. ${ }^{14}$ Current study aims at numerically investigating the self-starting capability of a VAWT using the immersed boundary projection method. ${ }^{15}$ In order to explore the parametric space in relatively short computational time and have more understanding of the physics of self-starting under a more extreme condition, flows are simulated at low Reynolds numbers and restricted to a two-dimensional cross section of an otherwise planar turbine geometry. A precise accounting for three-dimensional effects awaits future simulations. The motion of the VAWT is coupled with the aerodynamic forces exerted on the blades and a simple load model, which is linearly proportional to the turbine angular velocity, is used. A three-bladed VAWT with NACA0018 blades is started at various Reynolds numbers and density ratios. Comparisons of flow-driven and motor-driven VAWT are made with the water-tunnel experiments performed by Araya and Dabiri ${ }^{1}$ to validate the load model. A simple quasi-steady model is proposed to analyze the starting torque of a multi-bladed VAWT. The total torque and blade torque distributions are compared between VAWT with various number of blades. Based on the torque distributions of a single-bladed VAWT with various static pitch angles, an optimal pitch distribution is proposed.

\section{Simulation setup and numerical method}

Figure 1 shows the schematic of a VAWT with three NACA0018 airfoils as blades rotating with an angular velocity, $\Omega$, about point $O$ in a freestream with velocity, $U_{\infty}$, coming from the left. The azimuthal angle, $\theta$, is defined as the angle between the black blade and the vertical axis as illustrated and $\theta_{0}$ is the initial value of the azimuthal angle, i.e., $\theta_{0}=\theta\left(t^{*}=0\right)$. In order to simulate the starting of a VAWT, two-dimensional

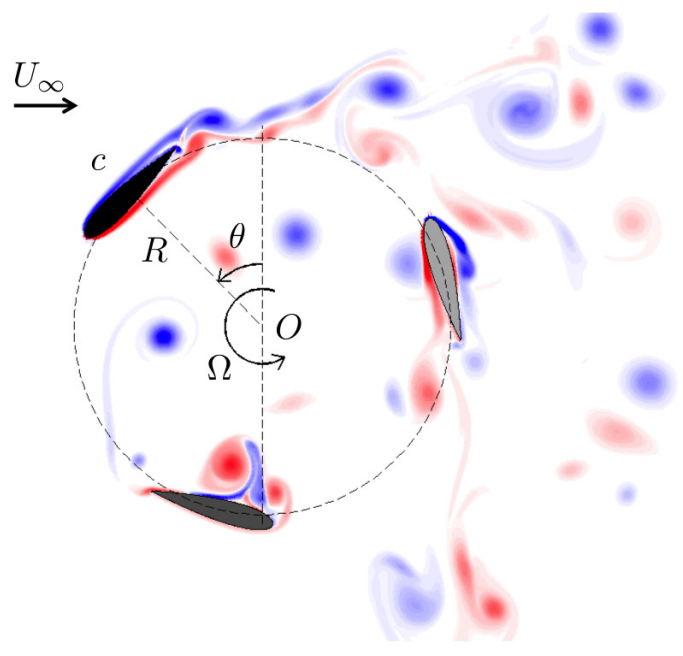

Figure 1. Schematic of a rotating three-bladed VAWT and the computational domain.

incompressible Navies-Stokes equations are coupled with the equation of the motion of the VAWT:

$$
N_{b} I_{O} \frac{d \Omega}{d t}=\tau_{f b}-\tau_{L}
$$

where $N_{b}$ is the number of the blades, $I_{O}$ is the moment of inertia of the blade about the rotation center of the VAWT, $\tau_{f b}$ is the moment exerted by the fluid about the rotation center of the VAWT, and $\tau_{L}$ is torque generated by a load. The moment of inertia of the blade, $I_{O}$, is obtained by computing the second moment 
of mass with respect to point $O$,

$$
I_{O}=\int_{V} \rho_{b}(\mathbf{x})|\mathbf{x}|^{2} d^{3} \mathbf{x}
$$

where $V$ is the volume of the blade, $\mathbf{x}$ is the position vector from point $O$ to a point in the blade, and $\rho_{b}(\mathbf{x})$ is the density field of the blade. $\tau_{f b}$ is determined by the aerodynamic forces exerted by the fluid on the blade, which behavior is complicated due to the inherently unsteady flow around the rotating blades and strong blade-vorticity interaction in the downwind half of a cycle. In this study, a simple load model:

$$
\tau_{L}=F \Omega
$$

is chosen, where $F$ is the friction coefficient of the load.

Four dimensionless parameters are introduced to systematically investigate the self-starting capability of a VAWT:

$$
\begin{aligned}
\text { tip-speed ratio: } & \lambda=\frac{\Omega R}{U_{\infty}}, \\
\text { solidity: } & \sigma=\frac{N_{b} c}{2 \pi R}, \\
\text { Reynolds number: } & R e=\frac{U_{\infty} c}{\nu}, \\
\text { density ratio: } & \phi_{\rho}=\frac{\rho_{e}}{\rho},
\end{aligned}
$$

where $R$ is the radius of the VAWT, $c$ is the chord length of the blade, $N_{b}$ is the number of blades, $\nu$ is the dynamic viscosity of the fluid, $\rho$ is the density of the fluid, and $\rho_{e}$ is the effective density of the blade, which is defined by

$$
\rho_{e}=\frac{\int_{V} \rho_{b}(\mathbf{x})|\mathbf{x}|^{2} d^{3} \mathbf{x}}{\int_{V}|\mathbf{x}|^{2} d^{3} \mathbf{x}}=\frac{I_{O}}{\int_{V}|\mathbf{x}|^{2} d^{3} \mathbf{x}} .
$$

The radius of the VAWT is fixed at $R=1.5 c$ in the present study in order to compare with experiments performed by. ${ }^{1}$ Therefore, the corresponding solidity is $\sigma=\frac{N_{b}}{3 \pi} \approx 0.106 \times N_{b}$ for VAWT with $N_{b}$ blades. From equation (1), we can see that when investigating the self-starting of a VAWT, the tip-speed ratio is a function of the other three parameters and the friction coefficient of the load, i.e.,

$$
\lambda=\lambda\left(R e, \phi_{\rho}, F, \sigma\right) .
$$

Two-dimensional incompressible flows in an airfoil-fixed reference frame are computed with appropriate forces added to the momentum equation to account for the non-inertial reference frame using the immersed boundary projection method developed by Colonius and Taira. ${ }^{15}$ The equation are solved on multiple overlapping grids that become progressively coarser and larger (greater extent). Equation (1) is coupled with the flow using a predictor-corrector method known as Heun's method. All ensuring computations use a $800 \times 800$ grid, which corresponds to $\Delta x=0.005$. The coarsest grid extends to 128 chord lengths in both the transverse and streamwise directions of the VAWT. The time step, $\Delta t$, is chosen to make Courant-Friedrichs-Lewy (CFL) number less than 0.4. VAWT are investigated numerically at low Reynolds number, $R e \sim O\left(10^{3}\right)$, in order to have more understanding of the starting of a VAWT in relatively short computational time. Although density ratio of the real VAWT is of $O(10)$, VAWT with low density ratios, $\phi_{\rho} \sim O(1)$, are investigated in order to explore the effect of density ratio on the starting of a VAWT at low Reynolds number.

\section{Results}

\section{A. Starting of a VAWT at various Reynolds numbers and density ratios}

We begin with starting a three-bladed VAWT with $\phi_{\rho}=1$ and $F=0$ from $\theta_{0}=0^{\circ}$ at $R e=500,1000,1500$, and 2000. Figure 2 shows the histories of the tip-speed ratio, which serves as the non-dimensionalized angular 
velocity, and the azimuthal angle. First, from figure 2(a), there exists a critical Reynolds number between 1000 and 1500 below which the VAWT does not start itself. Second, $\theta_{0}=0^{\circ}$ does not seem to be a favorable orientation for starting. At the beginning of the simulation, VAWT initially rotates in the opposite direction (clockwise) and then starts to rotate in the counter-clockwise direction, which is the correct direction given the orientation of the blades. Most interestingly, indicated by the dashed magenta and black lines in figure 2(b), we can see that the onset of counter-clockwise rotation commences when one of the blades is around $\theta=60^{\circ}$. On the other hand, VAWT that are unable to self-start oscillate around an equilibrium orientation with one of the blades is about $\theta=105^{\circ}$, which is indicated by the dashed blue and red lines in figure 2(b).. Further analysis on these behaviors using the starting torque are shown in section III.C.

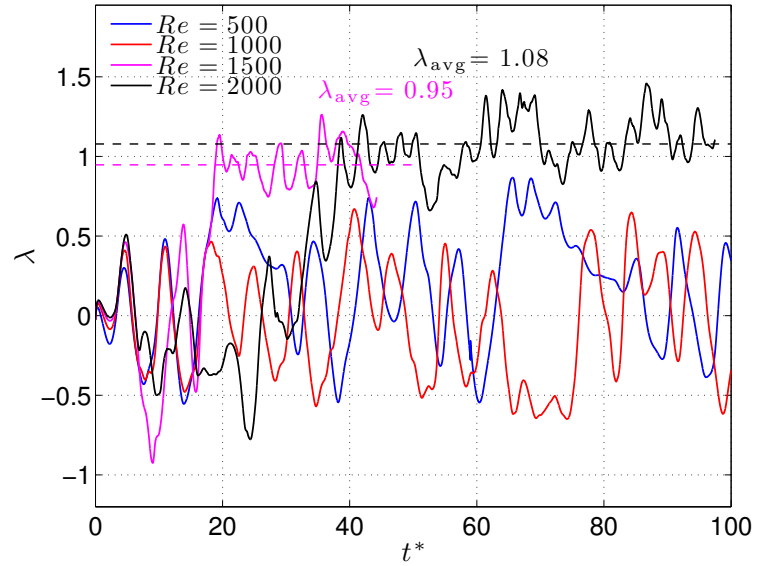

(a) Tip-speed ratio history.

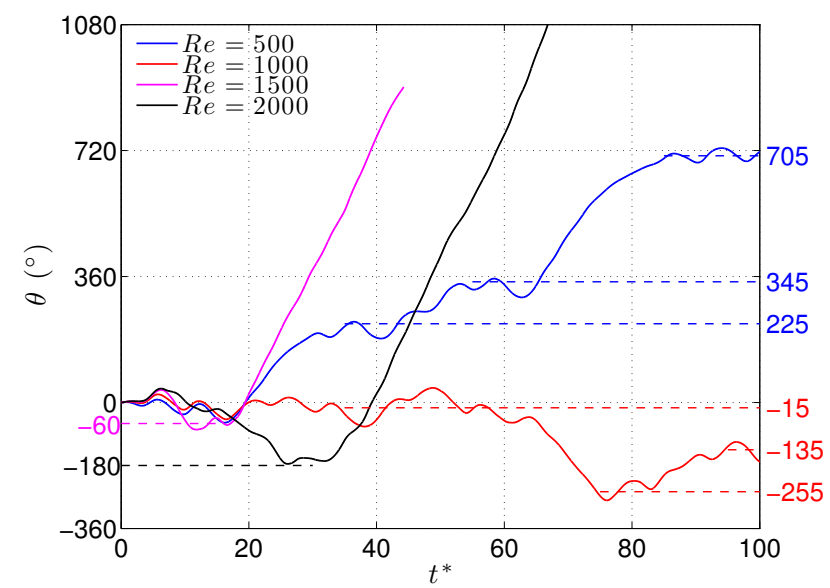

(b) Azimuthal angle history.

Figure 2. Histories of the tip-speed ratio and the azimuthal angle of a VAWT with $\phi_{\rho}=1$ and $F=0$ starting from $\theta_{0}=0^{\circ}$ at $R e=500,1000,1500$, and 2000 .

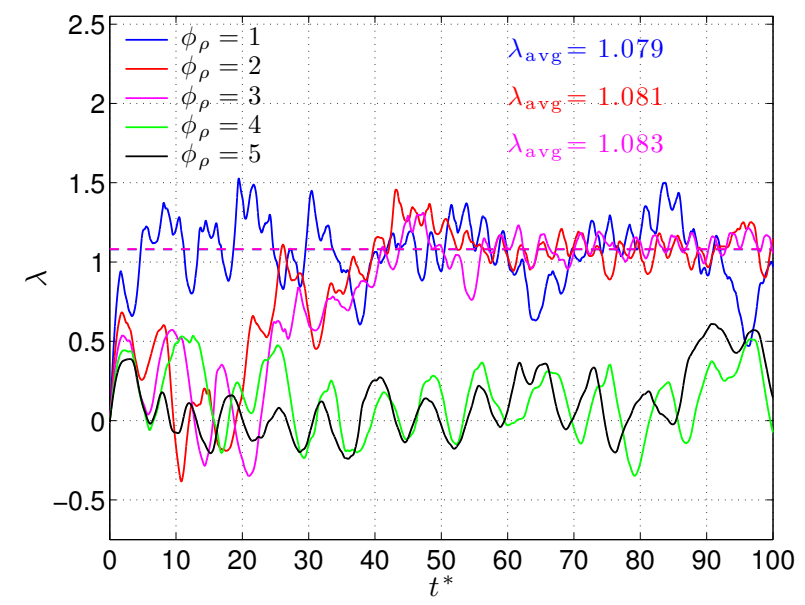

(a) Tip-speed ratio history.

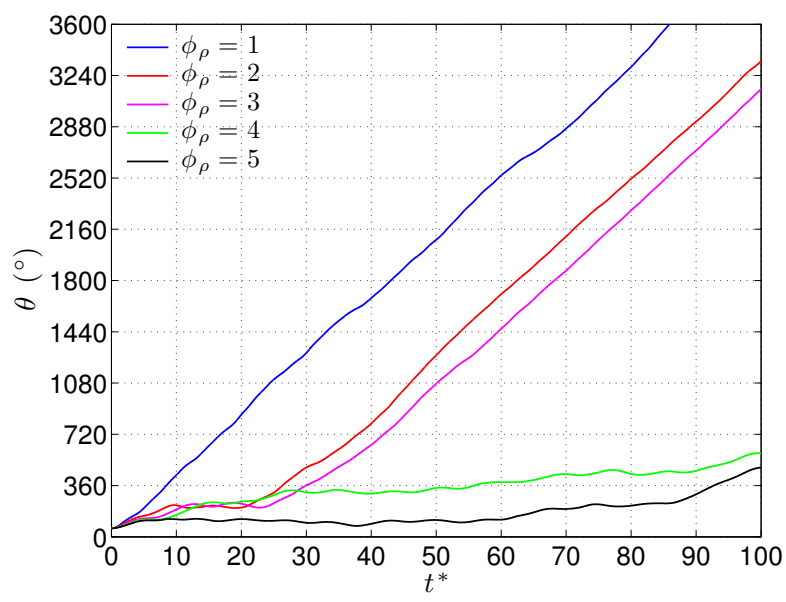

(b) Azimuthal angle history.

Figure 3. Histories of angular velocity and azimuthal angle of VAWT with $F=0$ and $\phi_{\rho}=1,2,3$, and 4 starting from $\theta_{0}=60^{\circ}$ at $R e=2000$.

We further investigate the three-bladed VAWT with $F=0$ and $\phi_{\rho}=1,2,3$ and 4 starting from $\theta_{0}=60^{\circ}$ at $R e=2000$. Figure 3 shows the corresponding tip-speed ratio and azimuthal angle histories. From figure $3(\mathrm{~b})$, we can see that when starting from $\theta_{0}=60^{\circ}$, VAWT that are able to self-start will rotate in the correction direction from the beginning. From figure 3(b), we observed a critical density ratio between 3 and 4 that VAWT with density ratio higher than the critical value is not able to self-start at $R e=2000$. When VAWT are able to self-start, VAWT with a lower density ratio reach stationary state faster but with a larger fluctuation in tip-speed ratio due to lower moment of inertia. Nevertheless, after they reach stationary state, 
the average tip-speed ratio of each VAWT are roughly the same. This fact indicates the averaged properties of the stationary state depend mainly on Reynolds number but not density ratio.

\section{B. Flow-driven VAWT and the comparison with motor-driven VAWT}

Based on the discussions in section III.A, the angular velocity of a VAWT driven by the flow, which can be characterized as a function of Reynolds number and density ratio, is determined by the aerodynamic forces exerted on the blades as in the field. However, prescribing the motion of a VAWT within a flow is common among both experimental and numerical investigations. In this section, we numerically investigate the difference between flow-driven and motor-driven VAWT by comparing power coefficients of flow-driven and motor-driven three-bladed VAWT. Moment coefficients are qualitatively compared with the torque of flow/motor-driven VAWT measured from experiments performed by Araya and Dabiri. ${ }^{1}$ The experiments were performed in a water tunnel at Reynolds numbers of $10^{4}$ and the setup of experiments are described in more detail in Araya and Dabiri. ${ }^{1}$

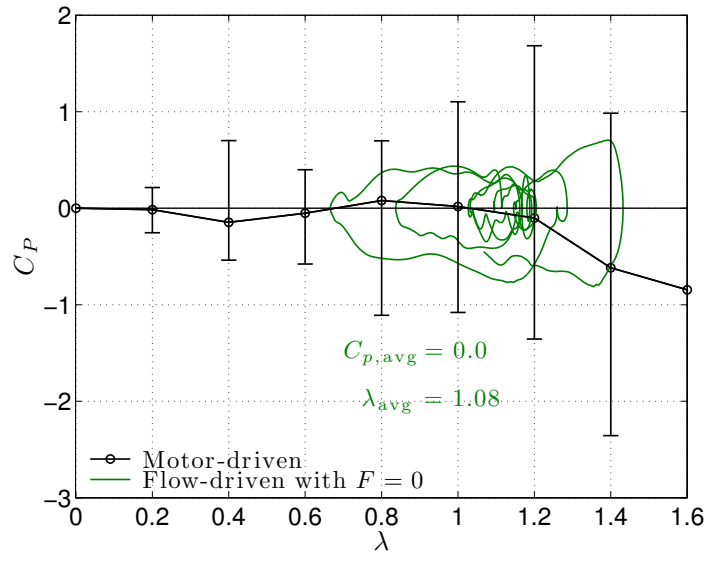

(a) $F=0$.

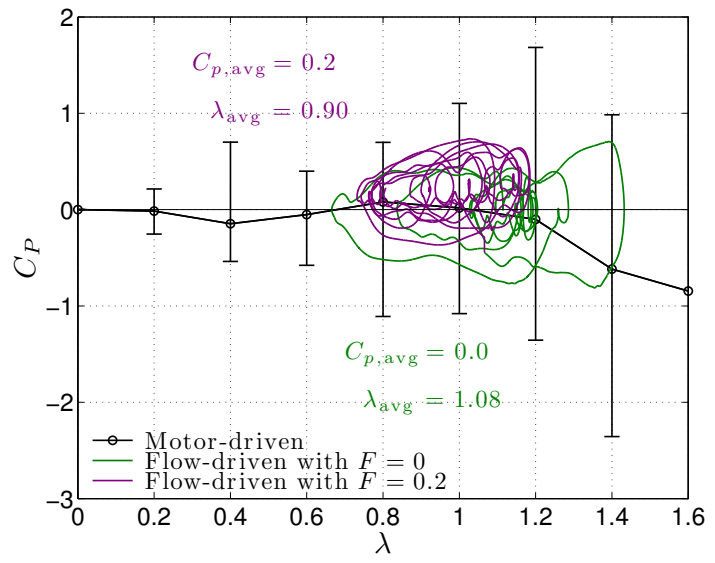

(c) $F=0$ and $F=0.2$.

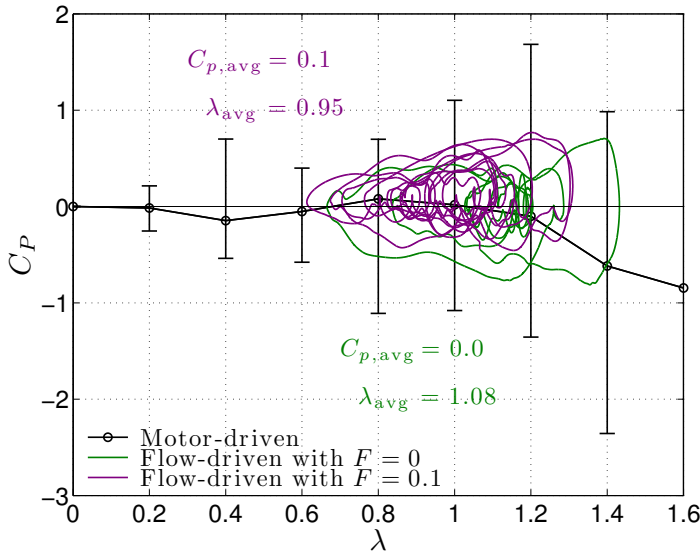

(b) $F=0$ and $F=0.1$.

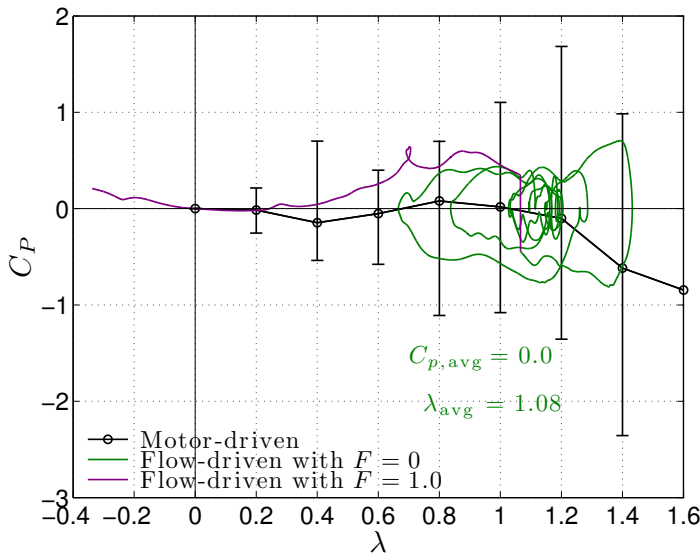

(d) $F=0$ and $F=1.0$.

Figure 4. Comparisons of power curves of a three-bladed VAWT motor-driven at various tip-speed ratios and flow-driven with $\phi_{\rho}=1$ and various friction coefficients at $R e=2000$.

Figure 4 shows the comparison of the power coefficients of a motor-driven VAWT at various tip-speed ratios and flow-driven VAWT with different friction coefficients at $R e=2000$. In each subfigure, we show the motor-driven power coefficient in black with the error bar showing the corresponding fluctuation in power coefficient at that tip-speed ratio and the power coefficient of a flow-driven VAWT with $F=0$ over three cycles in green. In figures 4(b), (c), and (d), the power curves of flow-driven VAWT with $F=0.1,0.2$, and 1.0 are shown in purple, respectively. The motor-driven power coefficient curve shows two equilibria at $\lambda \approx 0.7$ and $\lambda \approx 1.1$ where the VAWT generates zero power. Since the slope of the power coefficient curve 
is negative at $\lambda \approx 1$, it is a stable equilibrium. As expected, the flow-driven VAWT with $F=0$ generates almost zero power and is just free-spinning at an averaged tip-speed ratio $\lambda_{\text {avg }} \approx 1.08$. As $F$ increases, if the VAWT is able to rotate continuously, it operates at a lower averaged tip-speed ratio and generates a higher averaged power as shown in figures $4(\mathrm{~b})$ and $4(\mathrm{c})$. However, when $F$ is too large, say, $F=1.0$, the VAWT is unable to sustain rotation and the power output goes to zero as the turbine oscillates about a stable equilibrium with $\lambda \approx 0$ as shown in figure $4(\mathrm{~d})$.

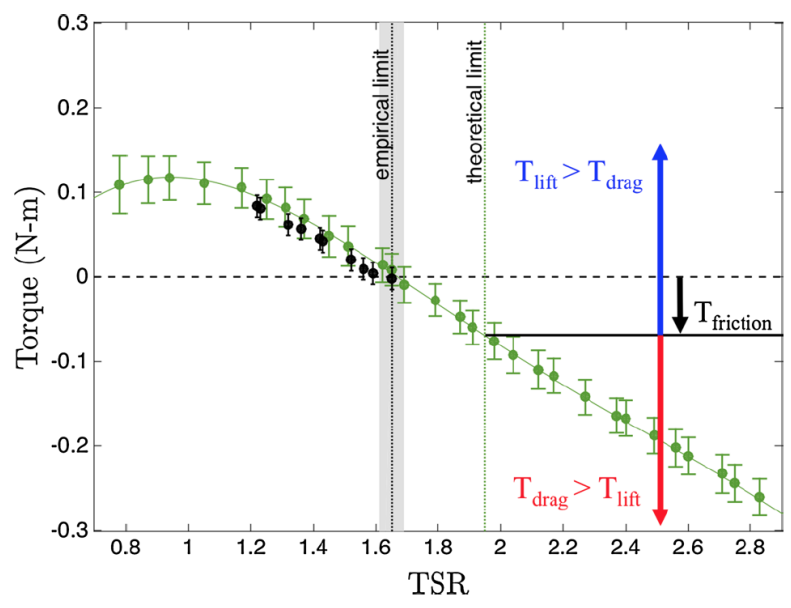

Figure 5. Measured torque for flow-driven (black) and motor-driven (green) cases. Error bars represent an estimate of one standard deviation in measurement error. A fourth-order polynomial is fit to the motor-driven points. Figure reproduced from Araya and Dabiri. ${ }^{1}$

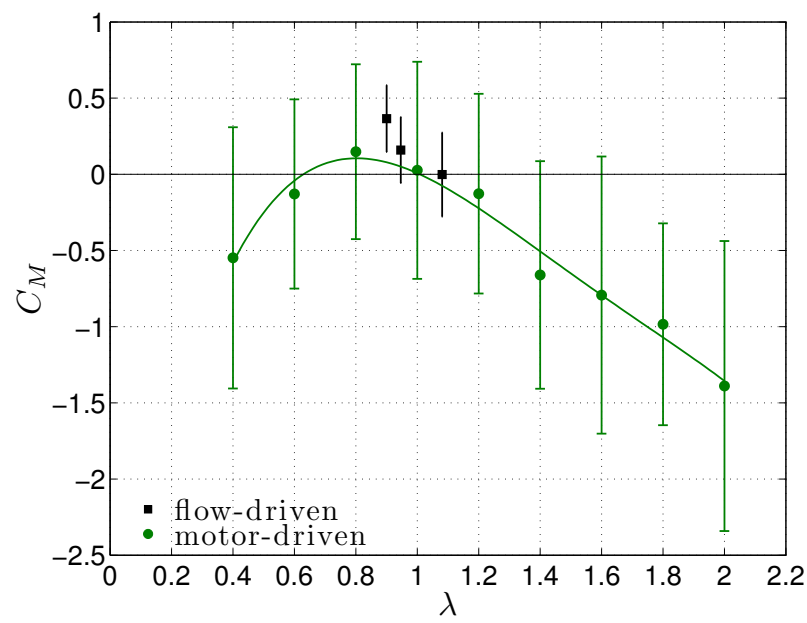

Figure 6. Moment coefficients for flow-driven (black) and motor-driven (green) cases. Error bars represent one standard deviation. A fourth-order polynomial is fit to the motor-driven points.

Figure 5, reproduced from Araya and Dabiri, ${ }^{1}$ presents turbine shaft torque measurements for a fixed $R e=2.6 \times 10^{4}$ and $\lambda$ ranging from approximately 0.9-2.8. There is qualitative agreement in the trend of the measured torque for similar flow-driven and motor-driven cases. Figure 5 also points out the theoretical limit of tip-speed ratio below which the difference between flow-driven and motor-driven wakes is minimal. Similarly, in figure 6 , we show the moment coefficients of flow-driven cases with $F=0,0.1$, and 0.2 and motor-driven cases for $\lambda$ ranging from $0.4-2.0$ at $R e=2000$. The flow-driven and motor-driven moment coefficients in the computation have good agreement between each other and similar behaviors to the torque for flow-driven and motor-driven VAWT measured in the experiments. These suggest that our load model is sufficient to represent the load of a flow-driven VAWT and a motor-driven VAWT can reproduce the physics of a flow-driven VAWT at $R e=2000$ when $\lambda$ is ranging from 0.4-2.0. 


\section{Analysis on the starting torque of a three-bladed VAWT}

The starting torque of a VAWT with various initial orientations are hard to compare directly due to their different rates of rotational acceleration. Therefore, we propose a simple model to analyze the starting torque. Consider the limit where the inertia of the VAWT is much larger than the inertia of the fluid so that the VAWT is barely rotating during starting. We can then treat the starting torque as the torque acting on a VAWT fixed in the flow.

We first simulate the flow past a three-bladed VAWT fixed at every five degrees. The flows start impulsively and the early torque distribution at $t^{*}=1$, which has not been affected by vorticity-blade interaction, is shown in the red curve in figure $7(\mathrm{a})$. Only the torques between $0^{\circ}$ and $120^{\circ}$ are shown for convenience because of the symmetry. The maximum of averaged torque occurs around $\theta_{0}=60^{\circ}$, which explains why $\theta_{0}=60^{\circ}$ is a favorable orientation to start a three-bladed VAWT. Two equilibria where the torque is equal to zero are found at $\theta_{0}=15^{\circ}$ and $\theta_{0}=105^{\circ}$. The equilibrium at $\theta_{0}=105^{\circ}$ is stable because the curve has a negative slope. As the VAWT slowly rotates away from $\theta_{0}=105^{\circ}$, the torque becomes stronger and pulls the VAWT back to the stable equilibrium. With similar arguments, equilibrium at $\theta_{0}=15^{\circ}$ is unstable, i.e., as the VAWT slowly rotates away from $\theta_{0}=15^{\circ}$, the increasing torque pushes the VAWT further away. Therefore, as shown in figure 2(b), VAWT that are not able to self-start oscillate around $\theta_{0}=105^{\circ}$.

Shown in figure $7(\mathrm{~b})$, we investigate the contributions of the torque from each blade to the starting torque for a three-bladed VAWT. For example, when VAWT is at $\theta_{0}=30^{\circ}$, its three blades are at $\theta_{0}=30^{\circ}$, $\theta_{0}=150^{\circ}$, and $\theta_{0}=270^{\circ}$. We plot the torque from each blade at the corresponding azimuthal angle in figure 7(b). These torque distributions of the blades are compared with the starting torque of a single-bladed VAWT. We can see that the torque distribution from each blade is close to that of a single-bladed VAWT except for a small difference in the downwind region $\left(180^{\circ} \leq \theta_{0} \leq 360^{\circ}\right)$. Due to the similarity of the torque distribution from each blade, the starting torque of a three-bladed VAWT can be well-reconstructed from the single-bladed torque distribution. The starting torque at a certain orientation is reconstructed by linearly combining the torques at the respective position of the blades. For instance, the torque of a three-bladed VAWT at $\theta_{0}=30^{\circ}$ can be reconstructed by adding the single-bladed torques at $\theta_{0}=30^{\circ}, \theta_{0}=150^{\circ}$, and $\theta_{0}=270^{\circ}$. The black curve in figure 7 (a) shows the reconstruction of the torque distribution from the single-bladed torque distribution obtained from figure 7 (b) (black curve). We can see that the reconstruction retains all important features of the three-bladed starting torque. The largest difference occurs at where the VAWT generates largest torque $\left(\theta_{0}=60^{\circ}\right)$.

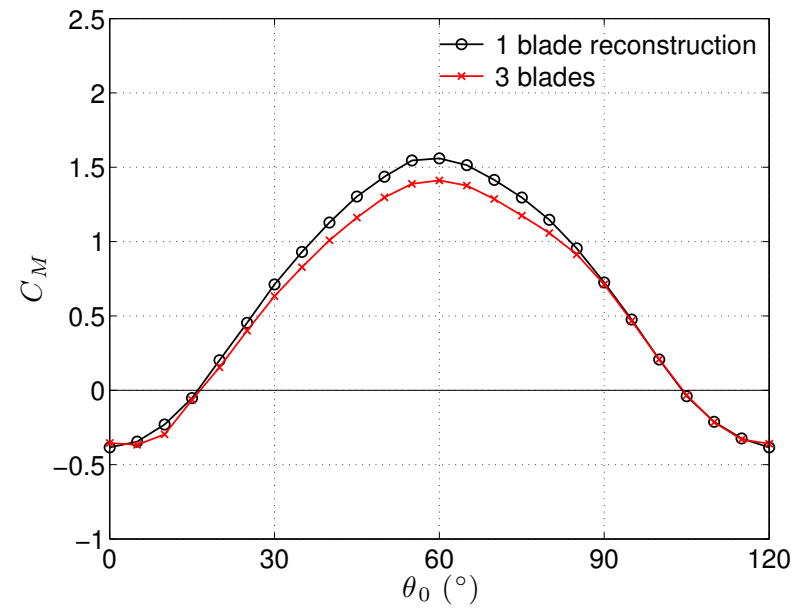

(a) Total torque.

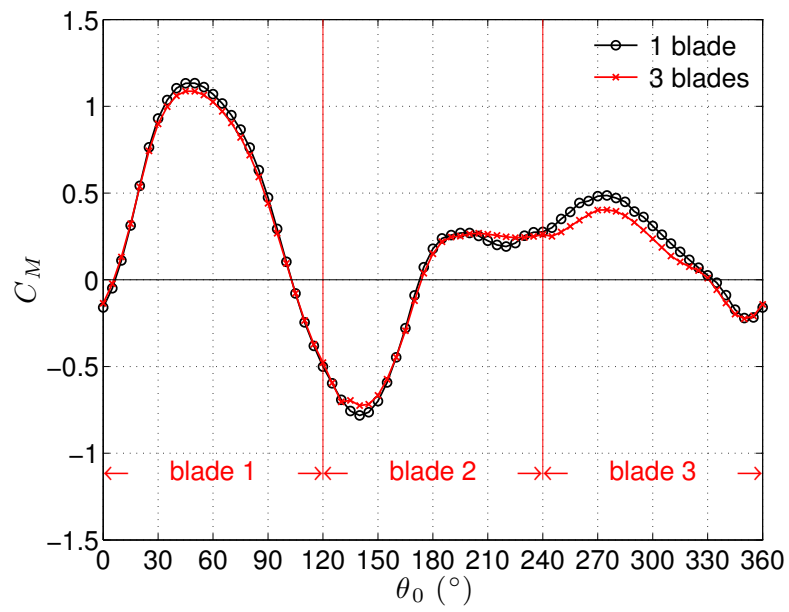

(b) Torque on each blade.

Figure 7. Torque distribution of a three-bladed VAWT at $t^{*}=1$ and $R e=2000$. 


\section{The starting torque distribution of a multi-bladed VAWT}

In order to investigate the effect of solidity on the starting torque, we investigate the torque of a multi-bladed VAWT fixed in the flow at various initial orientations as discussed in section III.C. Figure 8 shows the starting torques of VAWT with two to eight blades at $t^{*}=1$ and $R e=2000$. The torques of the multi-bladed VAWT with $N_{b}$ blades are shown only between $0^{\circ}$ and $\left(\frac{360}{N_{b}}\right)^{\circ}$ due to the symmetry. The range of initial orientations that generate a positive torque increase as the number of the blades increases. VAWT with more than three blades generate positive torque at all initial orientations. Moreover, we can again observe that stable equilibria for two and three-bladed VAWT exist around $\theta_{0}=115^{\circ}$ and $105^{\circ}$, respectively. Two, three, four, five, six, and eight-bladed VAWT generate the highest torques around $\theta_{0}=55^{\circ}, 60^{\circ}, 20^{\circ}, 30^{\circ}$, $20^{\circ}$, and $0^{\circ}$, respectively, which indicate the optimal orientations for starting.

Similarly, we also investigate the contributions of the torque from each blade to the starting torque for multi-bladed VAWT. These torque distributions of the blades are compared with the starting torque of a single-bladed VAWT in figure $8(\mathrm{~b})$. When the solidity is lower than 0.5 , the torque distribution from each blade is close to one of a single-bladed VAWT except for a small difference in the downwind region $\left(180^{\circ} \leq \theta_{0} \leq 360^{\circ}\right)$, highlighting the lack of vorticity-blade interactions amongst the different blades at early times. The average value of the torques over a cycle labelled in figure 8 increases as the number of the blades increases. However, when the solidity is larger than 0.5 , the torque distribution from each blade is different from one of a single-bladed VAWT. As the number of the blades increases, the discrepancy in the torque distribution increases and the average value of the torques over a cycle may decreases.

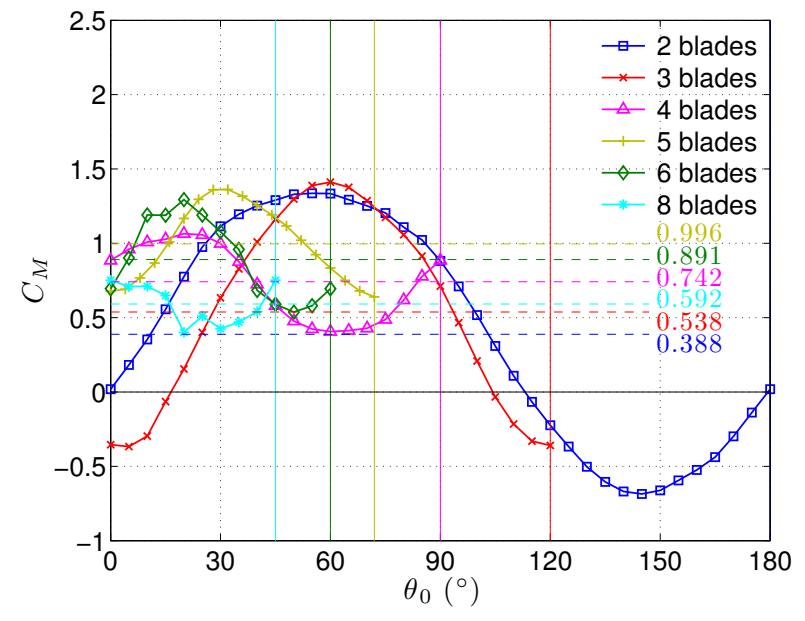

(a) Total torque.

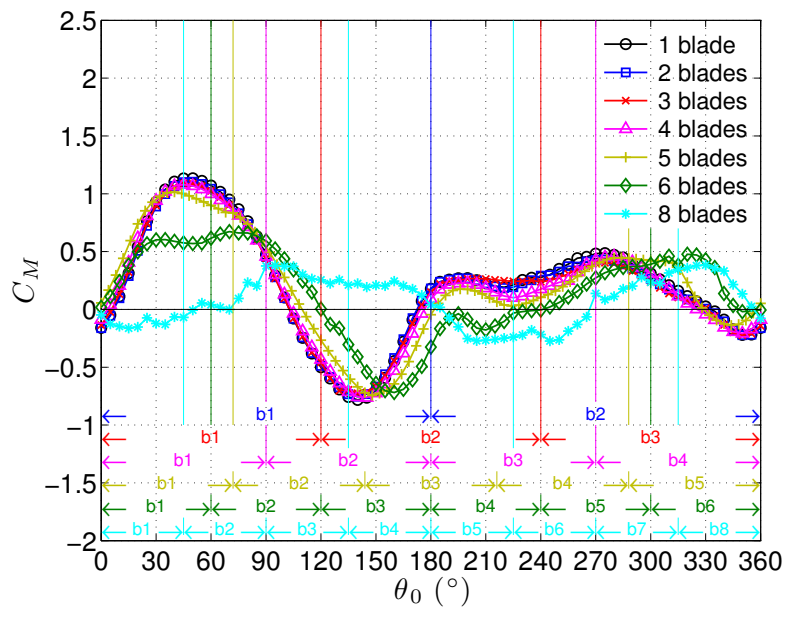

(b) Torque on each blade.

Figure 8. The torque distributions with $\sigma \approx 0.106$ (1 blade), 0.212 (2 blades), 0.318 (3 blades), 0.424 (4 blades), 0.637 ( 6 blades), and 0.848 ( 8 blades) at $t^{*}=1$ at $R e=2000$.

\section{E. Effect of the static pitch angle on the single-bladed torque distribution}

We showed in sections III.C and III.D that the multi-bladed starting torque can be well reconstructed from the single-bladed torque distribution when the solidity is lower than 0.5. Here we investigate the extent to which the static pitch angle can be changed to optimize the starting torque. As illustrated in figure 9(a), a constant static pitch angle is applied to the blade and the torque distribution changes correspondingly. The constant static pitch angle, $\psi$, is zero in the tangential direction, positive when the blade is rotated counter-clockwise to the turbine's center. Once the optimal static pitch angles at various azimuthal angles are obtained, the optimal starting configuration for a multi-bladed VAWT can be determined by pitching the blades with their own optimal static pitch angles as shown in figure 9(b).

The single-bladed torque distributions with various constant static pitch angles are examined in figure 10(a). The black dashed curve is the single-bladed torque distribution with zero pitch angle. The red and 


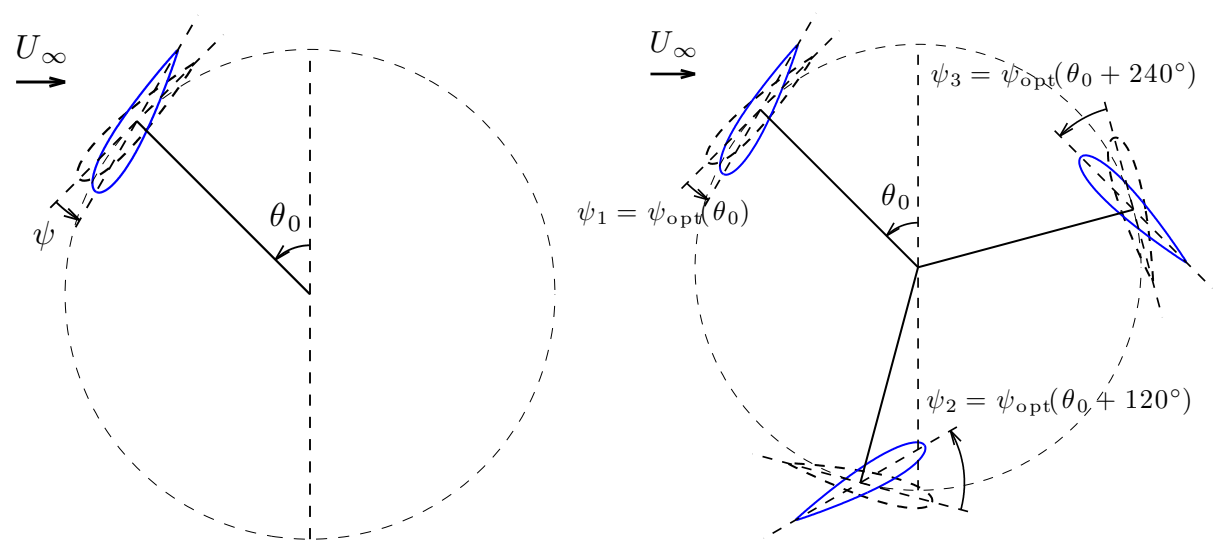

(a) A single-bladed VAWT with a static (b) Reconstructing the optimal three-bladed pitch angle. torque distribution from single-bladed results.

Figure 9. Single and three-bladed VAWT with static pitch angle(s).

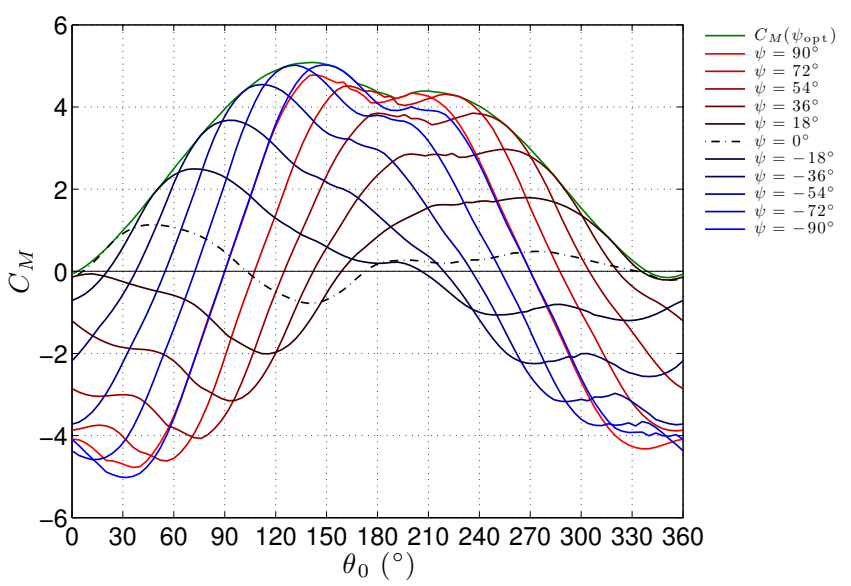

(a) Torque distributions with various static pitch angles (blue, black, and red curves) and the optimal torque distribution (green curve).

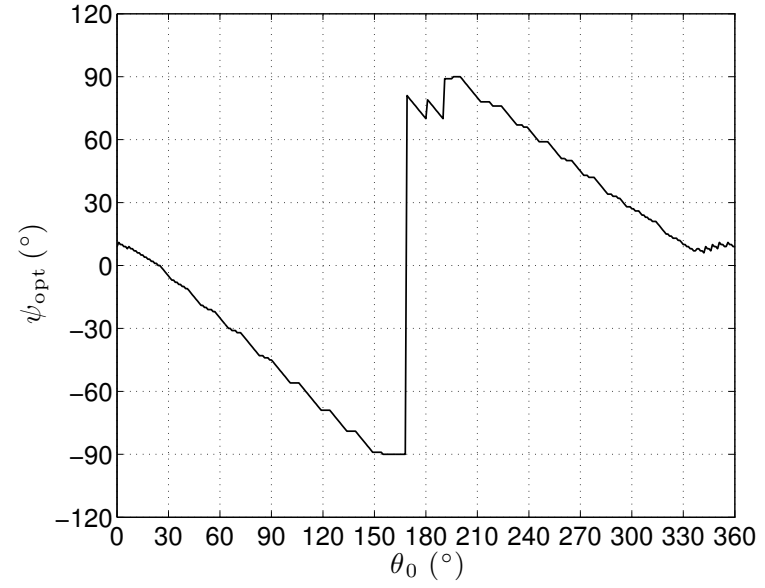

(b) The optimal static pitch angles.

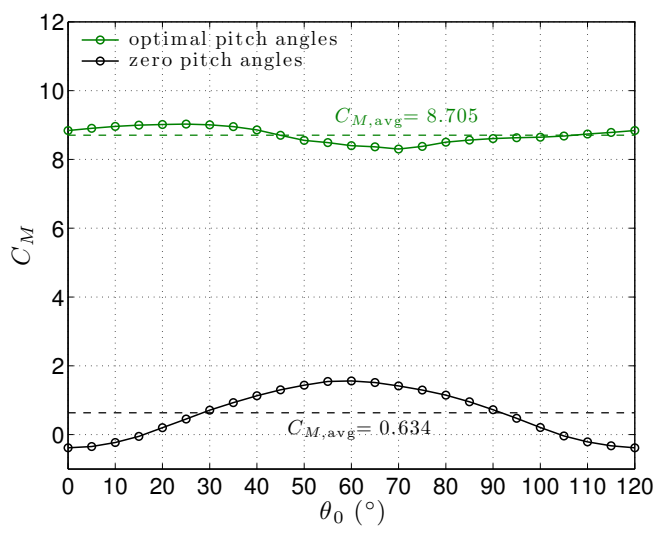

(c) Three-bladed torque distribution reconstructed by optimal single-bladed torque distribution.

Figure 10. Torque distributions of a single-bladed VAWT with various static pitch angles, the optimal static pitch angles, and the corresponding reconstruction of the three-bladed torque distribution. 


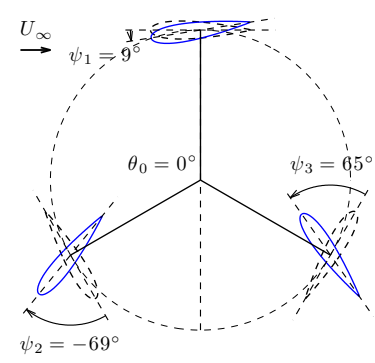

(a) $\theta_{0}=0^{\circ}$.

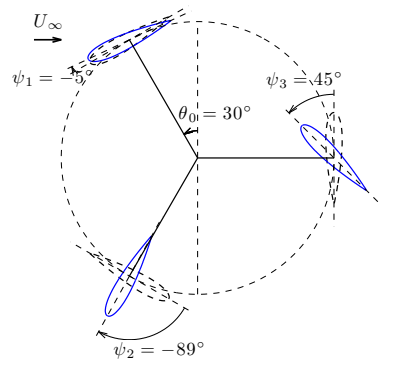

(b) $\theta_{0}=30^{\circ}$.

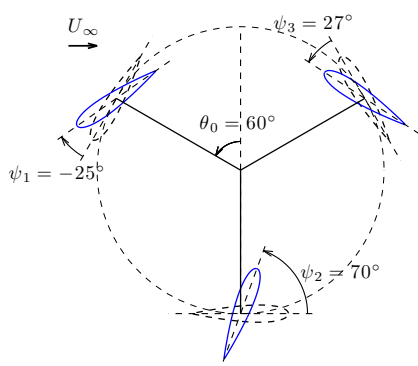

(c) $\theta_{0}=60^{\circ}$.

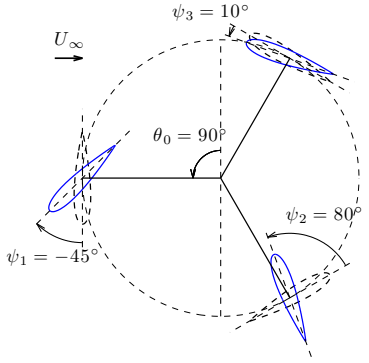

(d) $\theta_{0}=90^{\circ}$.

Figure 11. A three-bladed VAWT with optimal static pitch angles fixed at $\theta_{0}=0^{\circ}, 30^{\circ}, 60^{\circ}$, and $90^{\circ}$.

blue curves correspond to positive and negative pitch angles, respectively. We can see that at any initial azimuthal angle, there exists an optimal static pitch angle that generates the highest torque. The torque distribution corresponding to these highest (optimal) torques is the upper envelope of these curves and is shown as the green curve in figure 10(a). The corresponding optimal static pitch angles are shown in figure 10(b). The optimal three-bladed torque distribution can be reconstructed from the results shown in figure 10(a) using the procedure already illustrated in figure 9(b). For example, when a three-bladed VAWT is at $\theta=\theta_{0}$, its three blades are at $\theta=\theta_{0}, \theta_{0}+120^{\circ}$, and $\theta_{0}+240^{\circ}$. The optimal static pitch angles can be found from figure 10(b). We then sum up the torques at those azimuthal angles with their own optimal static pitch angles from figure 10(a). The resulting torque distribution is shown in the green curve in figure 10(c) and compared with the three-bladed torque distribution reconstructed from the single-bladed, zero pitch angles result. We can see that the three-bladed torques with optimal static pitch angles are positive and much higher than with zero pitch angles at all orientations. Moreover, figure 11 shows a three-bladed VAWT with optimal static pitch angles at $\theta_{0}=0^{\circ}, 30^{\circ}, 60^{\circ}$, and $90^{\circ}$ to give an idea how we can produce the largest torque at those orientations.

\section{Conclusions}

The starting capability of a VAWT was numerically investigated by simulating the flow around a freeto-rotate three-bladed VAWT. The predictor-corrector method was used to couple the motion of the VAWT with the aerodynamic forces exerted on the blade and shaft torque generated by a load. A simple load model, which is linearly proportional to turbine angular velocity, was used. Tip-speed ratio, which is associated with the angular velocity of the VAWT, was characterized as a function of Reynolds number and density ratio. Simulations showed that there exists a critical Reynolds number below which the VAWT cannot start itself. Flow at a higher Reynolds number drives the VAWT to rotate faster. At the same Reynolds number, VAWT with a density ratio lower than the critical value is capable of self-starting. A smaller density ratio results in a shorter time needed for VAWT to reach the stationary state but a larger fluctuation in angular velocity due to a smaller moment of inertia. Moreover, the averaged properties of the stationary state depend mainly on the Reynolds number but not on the density ratio.

The power outputs and the moment coefficients of motor-driven and flow-driven VAWT were compared. The flow-driven power coefficients lie within the power fluctuations of a motor-driven turbine at various tip-speed ratios. With a larger friction coefficient, the VAWT generates a higher averaged power output but operates at a lower averaged tip-speed ratio until the friction coefficient is too large for a VAWT to rotate at Reynolds number examined. Moreover, the flow-driven and motor-driven moment coefficients in the computation have good agreement between each other and are qualitatively similar to the torque measured in experiments performed by Araya and Dabiri. ${ }^{1}$ These computations suggest that the load of a flowdriven VAWT can be well-represented by the proposed load model and within the range of tip-speed ratio examined, a motor-driven VAWT can reproduce the physics of a flow-driven VAWT at low Reynolds number.

A simple model was proposed in order to analyze the starting torque of VAWT. The inertia of the blade is assumed to be much larger than the fluid, i.e., $\phi_{\rho} \gg 1$, so that the VAWT barely rotates and can be 
considered fixed in the flow. Analysis of the starting torque at early time indicates the two important orientations associated with the starting of a VAWT: orientation corresponding to the largest torque generation, at which a self-starting turbine always starts, and a stable equilibrium, where a non-self-starting turbine oscillates. These features agree with observations from the full simulations of the starting of a VAWT.

The effect of turbine solidity on the starting torque and the contribution to the starting torque from each blade for a multi-bladed VAWT were investigated. When solidity is smaller than 0.5 , the averaged starting torque over a cycle increases with solidity due to a growing range in generating positive starting torque and the multi-bladed torque distribution from each blade is close to the single-bladed one. The total torque distribution reconstructed from the single-bladed torque distribution retains important features. On the other hand, when solidity is above 0.5, the discrepancy between single and multi-bladed blade torque distributions grows and the averaged total torque over a cycle decreases as solidity increases. Therefore, for a sufficiently low turbine solidity of about 0.5 , the starting torque distribution can be modeled by considering a single blade at different orientations and constructed for multi-bladed turbines by linearly combining the torques at the respective positions of the blades. The torque distributions of the single-bladed VAWT with various static pitch angles were then investigated. An optimal pitch distribution was determined by the pitch angles that maximize the torque generation at individual orientation. Using this model, we found optimal starting configuration for a multi-bladed low-solidity VAWT.

\section{Acknowledgments}

This project is sponsored by the Caltech Field Laboratory for Optimized Wind Energy with Prof. John Dabiri as PI under the support of the Gordon and Betty Moore Foundation. We would like to thank Prof. John Dabiri and Dr. Daniel Araya for providing the experimental data and their advice on this research. We would also like to thank Prof. Beverley McKeon and Dr. Reeve Dunne for their helpful comments on our work.

\section{Reference}

${ }^{1}$ Araya, D. B. and Dabiri, J. O., "A comparison of wake measurements in motor-driven and flow-driven turbine experiments," Experiments in Fluids, Vol. 56, No. 7, 2015, pp. 1-15.

${ }^{2}$ Kirke, B. K., Evaluation of self-starting vertical axis wind turbines for stand-alone applications, Ph.D. thesis, Griffith University Gold Coast, 1998.

${ }^{3}$ Pawsey, N. C. K., Development and evaluation of passive variable-pitch vertical axis wind turbines, Citeseer, 2002.

${ }^{4}$ Baker, J., "Features to aid or enable self starting of fixed pitch low solidity vertical axis wind turbines," Journal of Wind Engineering and Industrial Aerodynamics, Vol. 15, No. 1, 1983, pp. 369-380.

${ }^{5}$ Hill, N., Dominy, R., Ingram, G., and Dominy, J., "Darrieus turbines: the physics of self-starting," Proceedings of the Institution of Mechanical Engineers, Part A: Journal of Power and Energy, Vol. 223, No. 1, 2009, pp. 21-29.

${ }^{6}$ Dominy, R., Lunt, P., Bickerdyke, A., and Dominy, J., "Self-starting capability of a Darrieus turbine," Proceedings of the Institution of Mechanical Engineers, Part A: Journal of Power and Energy, Vol. 221, No. 1, 2007, pp. 111-120.

${ }^{7}$ Sheldahl, R. E. and Klimas, P. C., "Aerodynamic characteristics of seven symmetrical airfoil sections through 180-degree angle of attack for use in aerodynamic analysis of vertical axis wind turbines," Tech. rep., Sandia National Labs., Albuquerque, NM (USA), 1981.

${ }^{8}$ Untaroiu, A., Wood, H. G., Allaire, P. E., and Ribando, R. J., "Investigation of self-starting capability of vertical axis wind turbines using a computational fluid dynamics approach," Journal of Solar Energy Engineering, Vol. 133, No. 4, 2011, pp. 041010.

${ }^{9}$ Beri, H. and Yao, Y., "Effect of camber airfoil on self starting of vertical axis wind turbine," J. Environ. Sci. Technol, Vol. 4, No. 3, 2011, pp. 302-312.

${ }^{10}$ Beri, H. and Yao, Y., "Numerical simulation of unsteady flow to show self-starting of vertical axis wind turbine using Fluent," Journal of Applied Sciences, Vol. 11, 2011, pp. 962-970.

${ }^{11}$ Chen, C.-C. and Kuo, C.-H., "Effects of pitch angle and blade camber on flow characteristics and performance of small-size Darrieus VAWT," Journal of Visualization, Vol. 16, No. 1, 2013, pp. 65-74.

${ }^{12}$ Tsai, H.-C. and Colonius, T., "Coriolis effect on dynamic stall in a vertical axis wind turbine," AIAA Journal, 2015, pp. $1-11$.

${ }^{13}$ Dunne, R., Tsai, H.-C., Colonius, T., and McKeon, B. J., "Leading edge vortex development on pitching and surging airfoils: A study of vertical axis wind turbines," Int. Conf. Wake Jets Separated Flows, Stockholm, Sweden, 2015.

${ }^{14}$ Dunne, R. and McKeon, B. J., "Dynamic stall on a pitching and surging airfoil," Experiments in Fluids, Vol. 56, No. 8, 2015, pp. 1-15.

${ }^{15}$ Colonius, T. and Taira, K., "A fast immersed boundary method using a nullspace approach and multi-domain far-field boundary conditions," Computer Methods in Applied Mechanics and Engineering, Vol. 197, No. 25, 2008, pp. 2131-2146. 